\title{
High-Throughput Phenotyping Platforms for Transgenic Plants in the Research and Product Development
}

\author{
Dong Yul Sung* \\ Trait Testing Program, Monsanto, 110 TW Alexander Dr, Research Triangle Park, NC 27709 USA
}

\begin{abstract}
The world population is projected to reach to 9.7 billion people by 2050. With increasing population and improving living standards, the demand for food is accelerating. In order to meet increasing demand for food while arable land and other resources are decreasing, agriculture needs all the tools available to sustainably increase crop yields. Development of effective genetically modified (GM) traits to protect crops from abiotic and biotic stressors is a critical aspect of sustainable yield improvement. Efficient identification of traits and rapid integration of the traits into commercial elite germplasm requires robust and rapid trait testing. Monsanto has developed numerous high-throughput phenotyping platforms to support rapid trait identification and integration. Selected phenotyping platforms will be reviewed to gain understanding of how they are utilized for trait phenotyping.
\end{abstract}

Keywords Phenotyping, Crop, High throughput, GM product development, transgenic

\section{INTRODUCTION}

It was not long ago that Danica May Camacho from the Philippines was declared by the United Nations as the 7 billionth baby born in the world. The world population growth has been keeping its pace and is expected to reach 9.7 billion by 2050. This poses a great challenge to world agriculture, not only to feed the world population but also to sustain or improve the quality of living that we have today. This challenge becomes even more daunting with decreasing arable land due to urban development and deforestation, not to mention depletion of water sources for agriculture. Development in emerging countries and changes in life styles also demand more food production. People managed a living with a handful of grains in the past, but now they want to enjoy high quality foods such as meat, processed foods that require much more inputs for production than grains do. Global warming also changes the types of crops that can grow in a particular region (Parmesan 2006). New pests are emerging due to climate change. The frequency of extreme weather is increasing every year (Parmesan 2006). Improvements from conventional breeding and agronomic practices alone might not be sufficient to cope with all of these challenges. It is imperative to preserve the earth and hand it over to the next generation in good shape so that they can also have same or better opportunities that we have for managing our lives.

Genetic and technology innovations over the years have contributed to yield increases in crops to meet the demand of increasing population and societal changes. Adaptation of hybrid corn exploiting heterosis catapulted yield increase in the early 1900s (Crow 1998). Introduction of high planting density, herbicides, insecticides, and fungicides all contributed to yield increase in the last decades (Smith et al. 1963; Miflin et al. 2000). Introduction of the herbicide glyphosate and subsequent introduction of glyphosate tolerant GM crop plants enabled very effective weed control that brought another leap in crop production (Sharma et al. 2002). However, in order to sustain social gains from past crop improvements such as reduction of malnutrition, increased food security, and greater selection of food choices, we have to maintain agricultural innovation.

Before the introduction of next generation sequencing

Received November 11, 2015; Revised November 18, 2015; Accepted November 18, 2015; Published November 30, 2015

*Comesponding author Dong Yul Sung, dan.sung@monsanto.com, Tel: +919-406-5717, Fax: +919-406-5757 
platforms, the bottle neck in plant research was in understanding genetic components of plant species. Now, with information from next generation sequencing platforms, one can now relate phenotypes or traits of a plant species to specific DNA polymorphisms (Mardis 2008). Because of this technology innovation, plant phenotyping has become the new bottle neck in plant research. Here, different types and utilities of high-throughput phenotyping platforms deployed in Monsanto for evaluation and advancement of products in the research and development pipeline are reviewed.

\section{Integrated technologies for future agriculture biotechnology solutions}

Agriculture biotechnology has been fueled by elite germplasm produced by conventional breeding, and by continual upgrades of insect and weed controlling transgenes including new field protection technologies such as Roundup Ready Xtend Crop System from Monsanto. Elite germplasm produced by breeding has been and still will be a major pillar of GM product development. However, in order to continually improve GM products to keep up with ever-increasing crop demands due to population increase and with emerging challenges due to climate changes, innovative technologies must be integrated. This will be critical for the success of any biotechnology company in the coming years.

Monsanto is a good example for integrating innovative technologies into GM products to further improve the products to meet and exceed future demands of crops by acquiring new technologies and collaborating with industry-leading companies. The Climate Corporation was a weather-data driven company that provides insurance to farmers based on climate prediction that can help farmers protect profits even in the case of adverse weather conditions such as drought and flood (McMahon 2011). Monsanto translated data acquisition and modeling capability of the Climate Corporation to benefit farmers by providing data-driven guides for crop cultivation to farmers along with GM seed products. Novozymes is an industry leading company on production of commercial enzymes, microorganisms, and pharmaceutical ingredients (www.novozymes.com). Novozymes and Monsanto formed the BioAg Alliance which brings together Novozymes' and Monsanto's capabilities within microbial discovery, development and production. The BioAg Alliance is dedicated to fundamentally enhancing the R\&D of microbial technology to significantly increase productivity of crops for the benefit of agriculture, consumers, the environment and society at large. Another good example is BioDirect technology that employs naturally occurring molecules and mechanism including RNAi to provide protection against pest or weeds. Monsanto is working on providing potential new options for disease, insect and weed control to farmers using this technology (www.monsanto.com/products). Development of GM products, integration of GM traits into elite germplasm, adoption of new technology all require phenotypic evaluation of transgenes, germplasm, technology to validate their presumed efficacies. Various phenotyping platforms are therefore central to success of the aforementioned research and development processes.

\section{Phenotyping tools in research and development pipeline in Monsanto}

A typical product development pipeline in an agriculture biotechnology company contains 5 key phases of development. Phase one focuses on discovery where potentially efficacious genes are discovered using various discovery tools. Phase two puts the focus on establishing gene efficacy by testing them in crops under controlled environment settings as well as in the field to measure desired traits. Phase three expands the efficacy testing with large-scale field testing and more germplasm. Phase three is also when the company starts to collect early stage data for regulatory submission. Phase four devotes much of the effort to introgress proven genes into commercial elite germplasm and to generate regulatory data on GM product safety. Phase five or the final stage focuses on submission of safety related documents for regulatory approval from key US agencies such as USDA, EPA, and FDA as well as regulatory agencies in key market countries. Among numerous phenotyping platforms Monsanto deploys for product and technology evaluation, RootArray technology in phase one, RootXpose technology in phase two, automated greenhouse and field testing in phase three, 
marker-assisted selection (MAS) and marker-aided back cross (MABC) are reviewed.

\section{RootArray technology}

RootArray technology is a real-time imaging system with micro-fluidics that allows non-invasive study of gene expression (Busch et al. 2012). One potential way of taking advantage of RootArray is novel promoter discovery and evaluation. Tissue-specific promoters and/or promoters inducible to provided stimuli can be effectively identified and evaluated.

In the RootArray system, small plant seedlings can be grown in micro chambers where different solutions or stimuli can be provided and gene expression can be monitored based on a marker gene expression driven by different types of promoters (Fig. 1). Therefore, it allows monitoring of gene expression dynamics over time and quantitatively assessing gene expression in living cells. Due to the ability of monitoring gene expression in living cells over time, RootArray provides gene expression information beyond a snapshot of gene expression that extracted mRNA is able to provide. It also eliminates potential artifacts of gene expression readouts due to degradation of mRNA or over-representation of certain species of mRNA during mRNA extraction and gene expression signal amplification processes. RootArray also allows in vivo monitoring of gene expression changes in responses to various stimuli provided into individual plants in micro-chambers using micro-fluidics.

\section{RootXpose technology}

One of key challenges to phenotype root traits is the root excavation process where much of fine root traits are inevitably lost. RootXpose eliminates the need for excavation by growing a plant in a transparent gel system where non-invasive phenotyping of fine root traits as well as major root traits is possible (Ingram et al. 2012) (Fig. 2). A similar system is developed and published by Dr. Kochian's laboratory at Cornell University as well (Clark et al. 2011). Efficacy of transgenes of interest for modifying root traits can be readily and accurately evaluated in a non-invasive and high-throughput manner

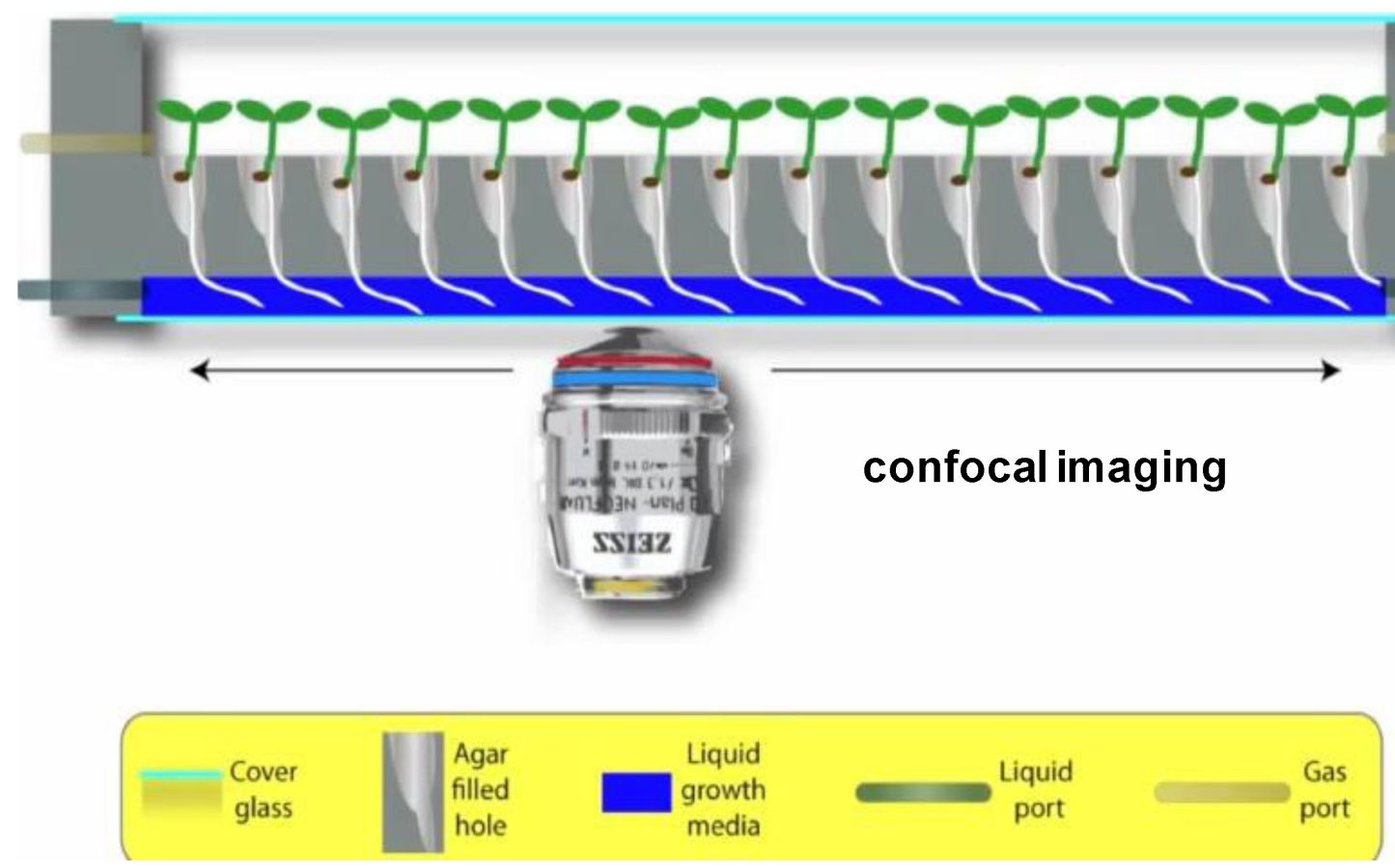

Fig. 1. RootArray system that allows in-vivo gene expression study of individual plant seedling. Small plant seedlings can be grown in micro chambers and different solutions or stimuli can be provided using micro-fluidics. Gene expression can be monitored based on a marker gene expression detected by non-invasive confocal imaging. 
for product advancement in the pipeline.

\section{Whole plant phenotyping using automated greenhouse}

Plant phenotyping at the whole plant level has been

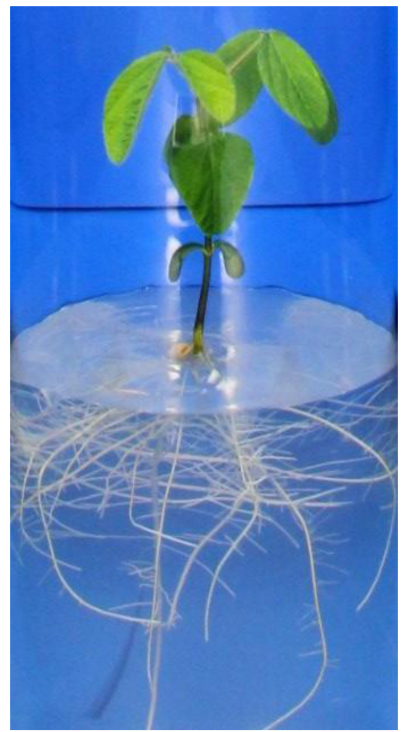

Fig. 2. Soybean grown in RootXpose system. Complexity of entire root system is available for three-dimensional phenotyping without need of excavation. typically conducted in small scale controlled environment settings or in the field with labor-intensive processes. In both cases, screening a large number of transgenes in a quantitative and high-throughput manner is a key challenge. An automated greenhouse at the Research Triangle Park (RTP) site (North Carolina, USA) overcomes this challenge by adopting industry-scale automated plant growth and proprietary imaging-based plant phenotyping. Key environmental and growth parameters such as temperature, photoperiods, light intensity, water level, and fertigation are programmable and tightly controlled. Plants grown in the automated greenhouse are moved through a set of instrumentation by a conveyor-belt system for image-based phenotyping and fertigation (Fig. 3). The imaging station is equipped with a set of visual and hyperspectral high resolution digital cameras in various angles to collect images of individual plants in a daily basis in order to capture morphological (biomass, plant height, canopy area) and hyperspectral traits (anthocyanin, chlorophyll, water content) of plants, then imaging analysis software automatically extracts traits of interest from each image for phenotyping.
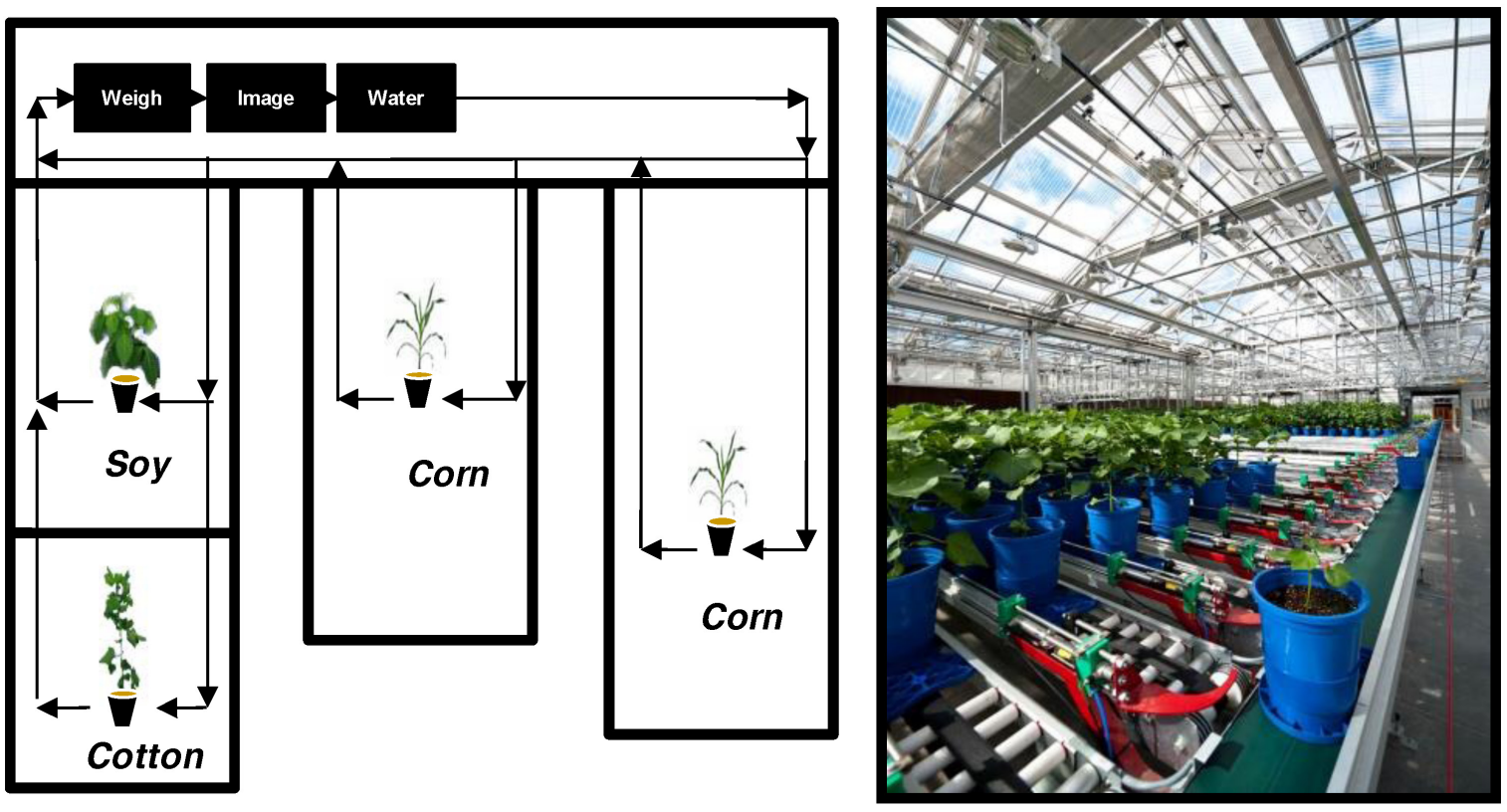

Fig. 3. Plants in the automated greenhouse are moved every day through a conveyor belt system for imaging-based phenotyping and automated fertigation. In each processing day, plants in the greenhouse bays are moved through conveyor belt system to get weighed, imaged, and watered automatically, then put back into the same greenhouse bay, but in a different position each time to normalize any potential micro-environment variations in the greenhouse bays. 
Automation of environmental control, plant growth, and phenotyping enables high precision phenotyping of GM plants in a high-throughput manner. One of the key advantages of automated greenhouse phenotyping is controlling water levels to evaluate gene efficacy for drought tolerance and nitrogen use efficiency. These two environmental parameters are often very hard to control in the field due to unpredictable weather conditions and various amount of residual nitrogen levels in the field. Effective screening of efficacious genes under controlled environment conditions such as automated greenhouse prior to expensive field testing is paramount to efficient product screening and development.

\section{Field testing of gene efficacy}

Field testing is the most critical phenotyping platform in the product development pipeline. A gene that has been proven to be efficacious in various phenotyping platforms must be proven that it still works under a wide range of field environment conditions including different regional weather conditions and soil types. In order to validate the efficacy of a transgene in a wide variety of field environment conditions, Monsanto utilizes its expansive testing network in the US and throughout the world (Fig. 4). After extensive field testing, only a handful of genes are validated for their efficacy and advanced to the next phase of product development.

\section{Marker-assisted selection (MAS) and marker-assisted backcrossing (MABC) for traits of interest}

A transgene that has been proven for its efficacy through various phenotyping platforms needs to be integrated into commercial elite germplasm. This is where biotechnology product development converges with the breeding pipeline to produce elite commercial germplasm. Rapid and efficient trait integration is crucial for successful product development. Combining multiple breeding traits in elite germplasm with various biotech transgenes is a big challenge. Each trait must have enough physical distance between them in the genome and a clear marker to track its presence in the genome to be efficiently integrated. Technology innovation such as next-generation sequencing platform has made genotyping with sequence polymorphism more affordable and faster than ever. In Monsanto, state of the art robotics is deployed in marker analysis processes than enables millions of plant marker analyses for breeders around the world (Fig. 5).

One of innovations in marker analysis in the company is
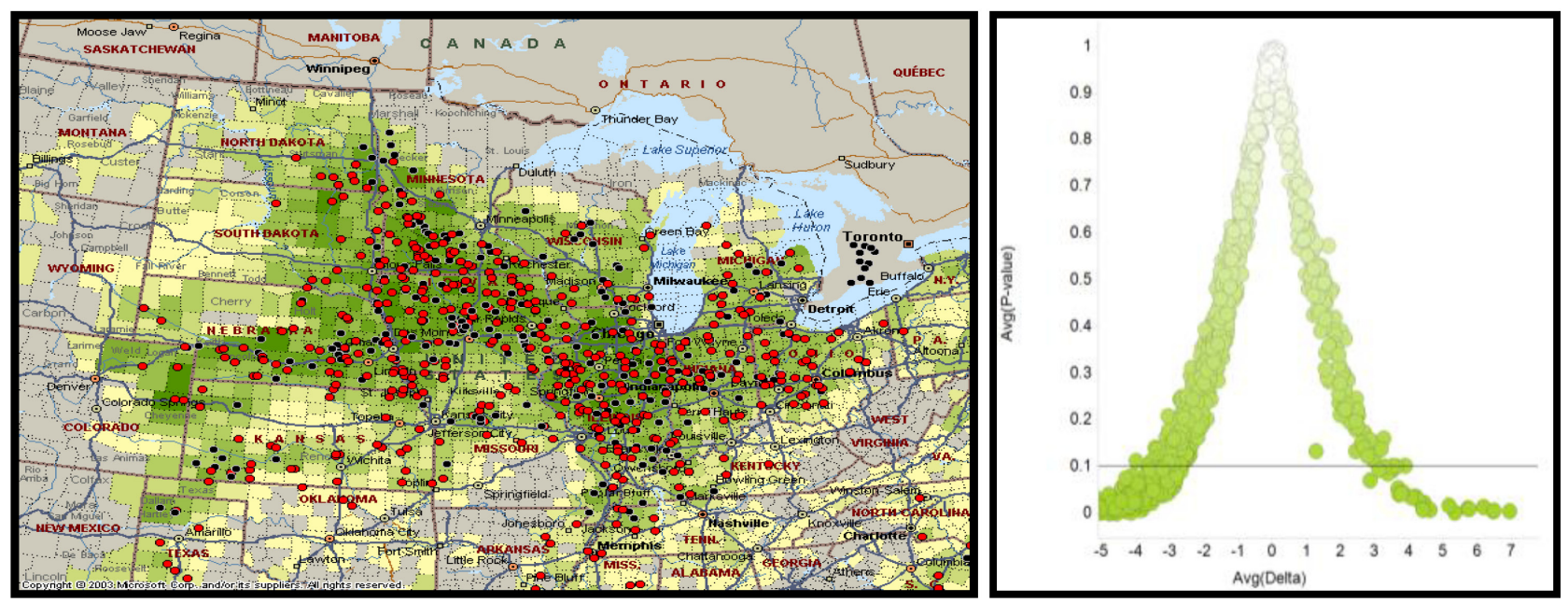

Fig. 4. Expansive testing geography enables effective product identification and advancement. Monsanto field testing networks in key US market regions are indicated as dots in the map and a "Volcano" graph of field testing outcomes demonstrates only a handful of genes pass through field testing and advance to next phase of development. 

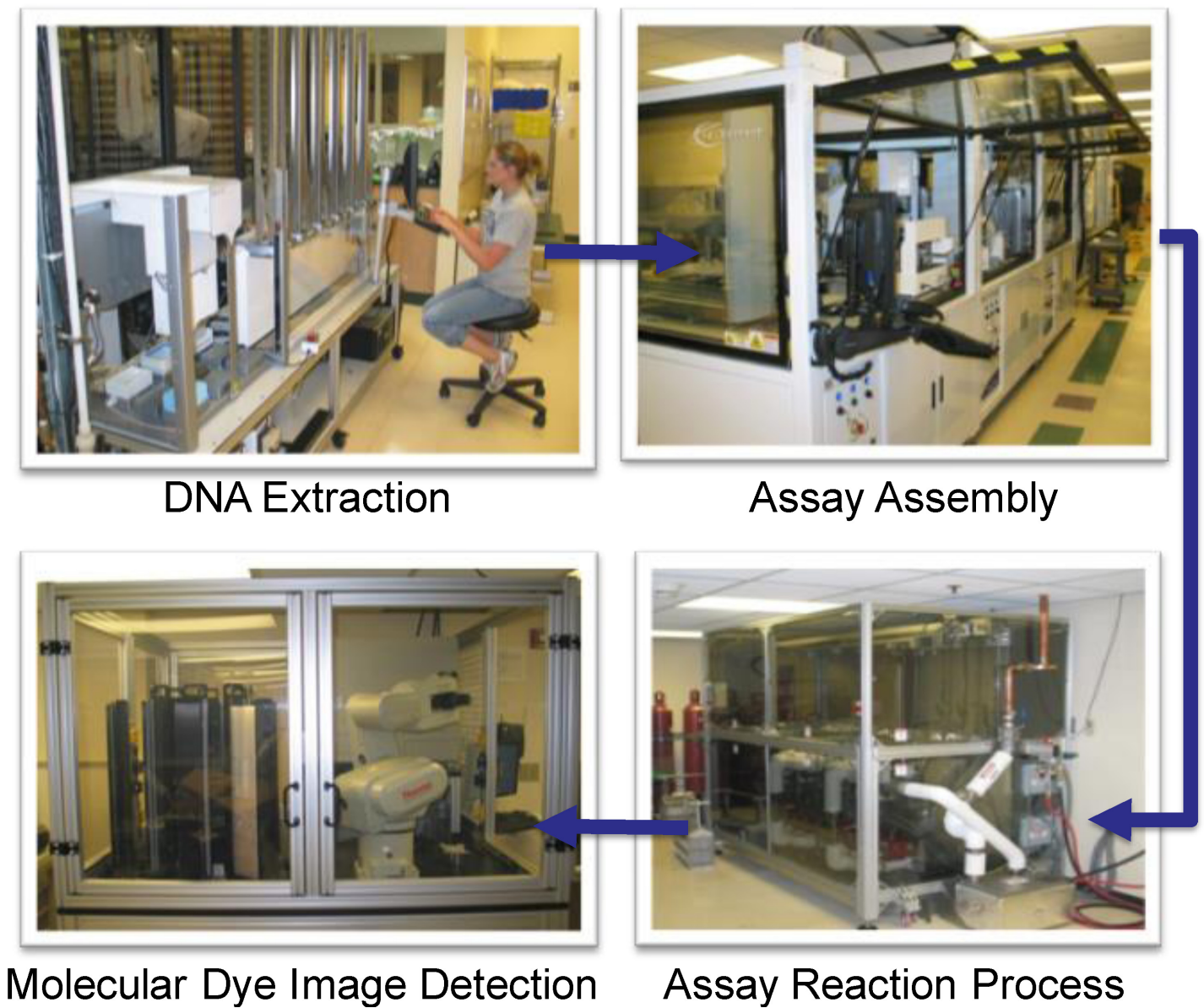

Fig. 5. State-of-the-art robotics delivers millions of plant DNA marker analyses to breeders around the world. A small amount of dried plant tissue material is deposited into each of 96 wells and processed for automated DNA extraction process, reagents for PCR reactions are automatically added in Assay Assembly, PCR reactions are carried out in multi-well formats in Assay Reaction Process, and PCR outputs are measured in dye intensity that is directly proportional to target sequence amplification in Molecular Dye Image Detection process.

seed chipping technology that allows genotyping prior to planting seeds in the field. Prior to implementation of seed chipping technology, segregating populations of transgenes had to be planted, tissue samples harvested, genotyping conducted, plant lines with traits identified, and other undesired plants thinned before desired plants are propagated in the field for seed production. Marker-Assisted Selection (MAS) with chipped seed materials allows skipping these time-consuming and labor intensive processes and directly going to plant propagation for seed production (Fig. 6).

Conversion or trait integration into commercial elite germplasm requires multiple rounds of backcrossing over several years. Cost-effective high throughput marker development processes facilitate identification of markers close to the location of trait of interest in the genome. With this marker and genotyping tools, the trait integration process has significantly shortened, saving resources and time required for product development. Marker-Assisted Backcrossing (MABC) brought a huge impact to Monsanto's product development by saving 1-2 years of backcrossing compared to traditional trait integration processes and by increasing success rates of trait integration by increasing percentage of recurrent parent genome in backcrossed populations (Fig. 7). 


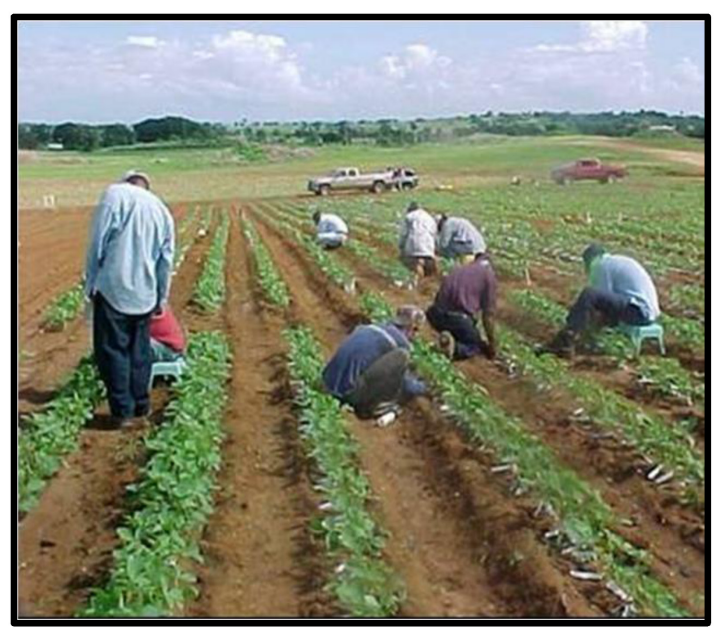

Genotyping using leaf samples

$>$ Labor and land intensive

$>$ Time-consuming

$>$ Low-throughput

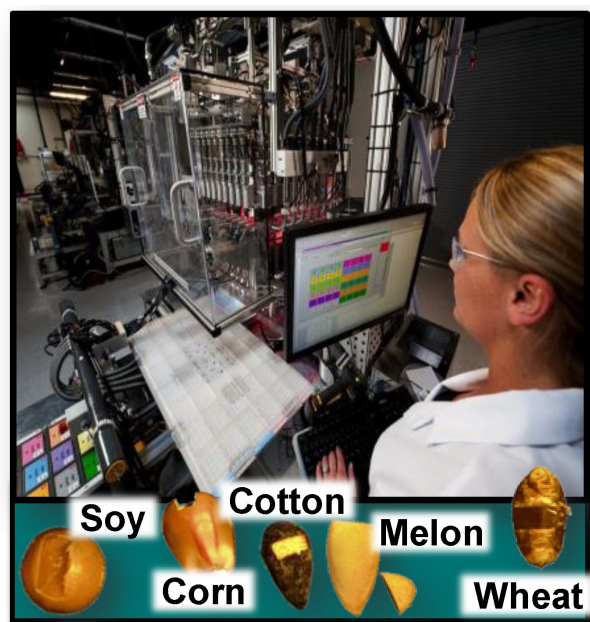

Genotyping using seed chipping

Capable of analyzing millions of samples per year with few errors

Select and plant only desired seeds!

Fig. 6. Automated Seed Chipping Removes the Bottleneck of Hand Sampling Plant Tissue for genotyping.
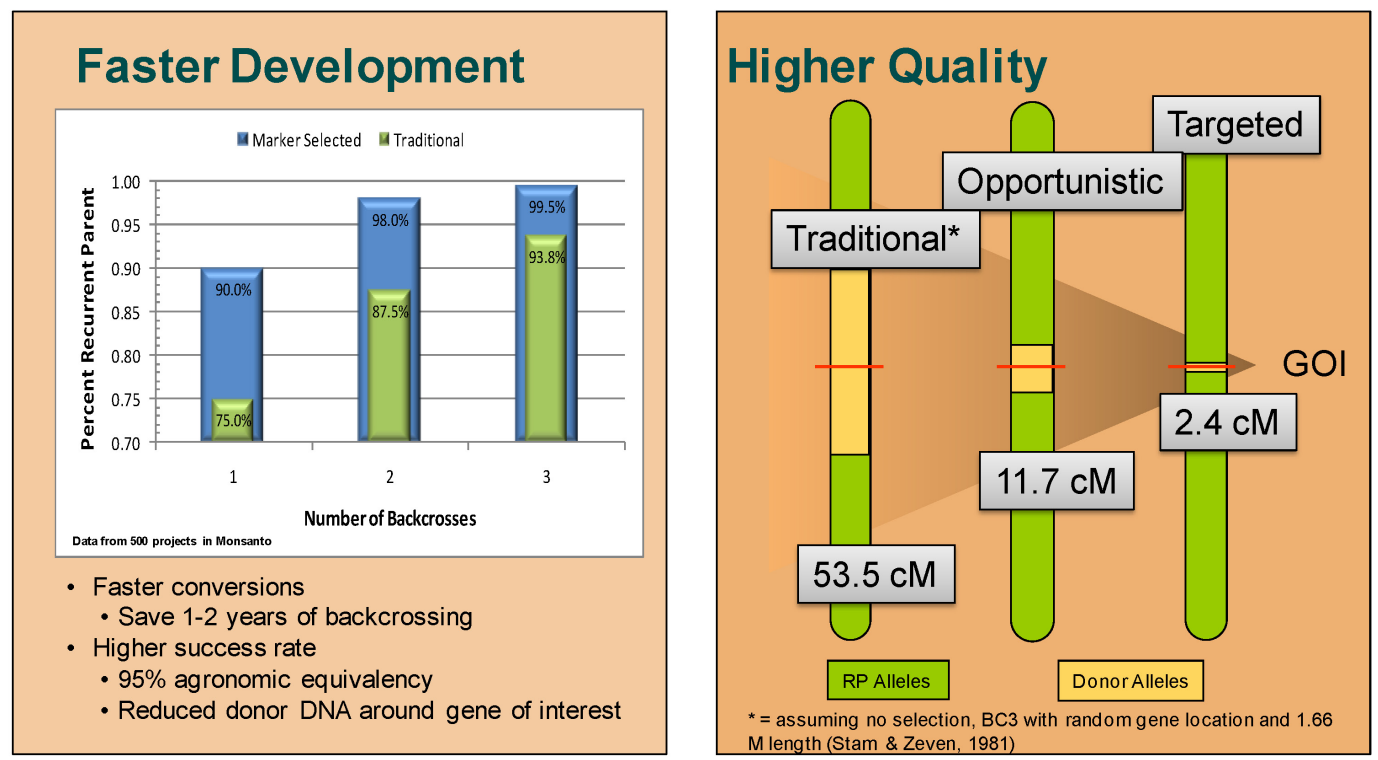

Fig. 7. Marker-Assisted Backcrossing (MABC) accelerates the integration of traits into elite germplasm. Marker assisted selection allows faster product development by selecting progenies with associated markers and quickly enriches recurrent parent genome in the backcrossed progenies to achieve higher quality.

\section{CONCLUDING REMARKS}

Agricultural biotechnology has contributed to food production increase to meet the need of growing popu- lations in developing countries. However, the need for increasing crop production is more challenging than ever with population growth, societal and diet changes in developing countries, climate change, and decreasing 
arable land around the world. For successful development of sustainable crop production, innovation and integration of new technology is essential. Monsanto is continually identifying and adopting new technology into its integrated product development platform. A few cases of new and existing phenotyping platforms in Monsanto's product development were reviewed to highlight the importance of phenotyping platforms for product development in the post-genome sequencing era. Integration of effective and efficient phenotyping platforms for trait identification and validation are key components not only for the success of production development in the company, but also for continual innovation of crop production to meet the demand of growing population in the world.

\section{ACKNOWLEDGEMENTS}

The author gives special thanks to Dr. Paul Chomet, Dr. Sam Eathington, Jee Jung, Dr. Tom Osborn, Chris Tierney, and Monsanto Communication for providing figure materials and to David Lanzotti, Drs. Donald Nelson and Eric Sachs, and Steven Screen for providing their expertise to the manuscript.

\section{REFERENCES}

Busch W, Moore BT, Martsberger B, Mace DL, Twigg RW, Jung J, Pruteanu-Malinici I, Kennedy SJ, Fricke GK, Clark RL, Ohler U, Benfey PN. 2012. A microfluidic device and computation platform for high-throughput living imaging of gene expression. Nat. Methods. 9:
1101-1106.

Clark RT, MacCurdy RB, Jung JK, Shaff JE, McCouch SR, Aneshansley DJ, Kochian LV. 2011. Three-Dimensional Root Phenotyping with a Novel imaging and Software Platform. Plant Physiol. 156: 455-465.

Crow JF. 1998. 90 Years Ago: The Beginning of Hybrid Maize, Genetics. 148: 923-928.

Smith WW, Betran J, Runge ECA. 1962. Corn; Origin, History, Technology, and Production. John Wiley \& Sons, Inc., Hoboken, NJ.

Ingram PA, Zhu J, Shariff A, Davis IW, Benfey PN, Elich T. 2012. High-throughput imaging and analysis of root system architecture in Brachypodium distachyon under differential nutrient availability. Philos. Trans. R. Soc. Lond. B. Bio. Sci. 367: 1559-1569.

Mardis ER. 2008. The impact of next-generation sequencing technology on genetics. Trends in Genetics. 24(3): 133-141.

McMahon K. 2011. The Climate Corporation offers precise weather insurance coverage. Farm Industry News Nov 3, 2011.

Miflin B. 2000. Crop improvement in the 21st century. J. of Exp. Bot. 51: 1-8.

Parmesan C. 2006. "Ecological and Evolutionary Responses to Recent Climate Change." Annu. Rev. Ecol. Evol. Syst. 37: 637-669.

Sharma HC, Crouch JH, Sharma KK, Seetharama N, Hash CT. 2002. Application of biotechnology for crop improvement: prospects and constraints. Plant Sci. 163: 381-395.

Stam P and Zeven AC. 1981. The theoretical proportion of the donor genome in near-isogenic lines of self-fertilizers bred by backcrossing. Euphytica. 30: 227-238. 